\title{
Pulmonary sequestration: a rare cause of false-positive uptake in lodine-131 whole-body scan
}

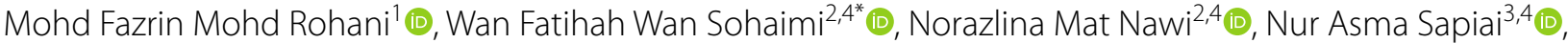 \\ Muhammad Azizul Mohd Daud ${ }^{4}$ (10 and Wan Hazirah Wan Sohaimi ${ }^{5}$
}

\begin{abstract}
Background: High-dose radioactive iodine-131 (I-131) is generally used post-total or subtotal thyroidectomy in differentiated thyroid cancer (DTC) patients. I-131 whole-body scan (WBS) is performed to localize I-131 accumulation in the body and for restaging after therapy. Nonetheless, there are numerous causes of false-positive uptake in I-131 WBS in the absence of residual thyroid tissue or thyroid cancer metastasis.

Case presentation: We present a case of 51-year-old lady with false-positive uptake in I-131 WBS mimicking lung metastasis. Patient underwent total thyroidectomy, and histopathological examination showed well-differentiated papillary thyroid microcarcinoma. Diagnostic l-131 WBS followed by two subsequent high-dose radioiodine ablation showed persistent I-131 uptake in the left lower lung field. However, the serum thyroglobulin (Tg) is incessantly undetectable $(<0.04 \mu \mathrm{g} / \mathrm{ml}$ ) with negative anti-thyroglobulin antibody (anti-TgAb). Three serial CT scans over the course of treatment coupled with MRI of the thoracolumbar region revealed stable lung lesion in the posterior segment of left lower lobe which signifies pulmonary sequestration. Based on the clinical assessment, imaging findings and blood investigations, patient was no longer subjected to I-131 therapy and is maintained on TSH suppression with L-thyroxine. After due consideration of the pros and cons of surgical intervention, the patient opted against surgical removal of the pulmonary sequestration.
\end{abstract}

Conclusions: Meticulous assessment of patient with suspected disease recurrence or metastasis in thyroid carcinoma is essential to avoid unnecessary I-131 ablation or surgical intervention. In this case, the positive I-131 imaging is associated with undetectable serum $\mathrm{Tg}$ (with negative anti-TgAb). Albeit uncommon, pulmonary sequestration should be taken into consideration as one of the differentials in false-positive findings of an l-131 WBS.

Keywords: lodine-131, Radioiodine, Pulmonary sequestration, Thyroid cancer

\section{Background}

Iodine-131 (I-131) has been used effectively over the years in both diagnosis and therapy of differentiated thyroid cancer (DTC). Whole-body scan (WBS) of I-131 from either diagnostic or therapeutic doses can reveal

\footnotetext{
*Correspondence: fatihahkk@usm.my

${ }^{2}$ Department of Nuclear Medicine, Radiotherapy and Oncology, School of Medical Sciences, Universiti Sains Malaysia, 16150 Kubang Kerian, Kelantan, Malaysia

Full list of author information is available at the end of the article
}

foci of normal residual thyroid tissue, carcinoma or remote metastasis in post-surgical DTC patients [1]. We report a case of DTC with persistent I-131 pulmonary uptake in the serial diagnostic and therapeutic WBS despite undetectable serum thyroglobulin (Tg). Although I-131 WBS is specific, there are myriad of abnormal lesions which can produce false-positive imaging results [1]. As for this case, radiological imaging advocates pulmonary sequestration (PS) as the main differential of persistent I-131 uptake in the left lower lung field. Although the diagnosis is not histopathologically proven, there 
are few articles which discussed the radiological imaging findings indicating the diagnosis $[2,3]$. The precise interpretation of I-131 WBS is important to avoid unnecessary high-dose I-131 ablation and to optimize the management of DTC as well as other non-thyroidal lesions.

\section{Case presentation}

A 51-year-old lady presented with progressive anterior neck swelling in March 2011. Total thyroidectomy was performed, and histopathological examination revealed a right thyroid lobe nodule $6 \mathrm{~mm}$ in size, that was compatible with well differentiated papillary thyroid microcarcinoma. Post-operative serum Tg was $0.09 \mu \mathrm{g} / \mathrm{ml}$, and the value of anti-thyroglobulin antibody (anti-TgAb) was $10.9 \mathrm{kIU} / \mathrm{L}$. She was risk stratified into the low-risk group (in view of microcarcinoma, low serum $\mathrm{Tg}$ and negative anti-TgAb) and based on the 2009 American Thyroid Association (ATA) guidelines, consideration for pretherapy diagnostic WBS is only recommended if a change of management is to be expected [4]. As the patient was in the low-risk group, levothyroxine was commenced for thyroid-stimulating hormone (TSH) suppression with concurrent serial serum $\mathrm{Tg}$ and anti-TgAb monitoring.

Three years later, she presented to the hospital with a chief complaint of haemoptysis. CT thorax (2.12.14) showed an ill-defined heterogeneously enhancing mass with spiculated margin at the posterobasal segment of left lower lobe (measuring $4.1 \times 2.0 \mathrm{~cm}$ ) suspicious of malignancy (Fig. 1). Subsequently, another two serial CT scans over a period of 1 year were performed in addition to an MRI of the thoracolumbar region with findings showing stable lung lesion in the posterior segment of left lower lobe that signifies pulmonary sequestration (Fig. 2). Both imaging modalities advocate pulmonary sequestration as the main differential.

Diagnostic I-131 WBS was subsequently ensued on 1.11.15 and demonstrates two foci of intense increased tracer uptake in the thyroid bed with accompanying star artefact as well as I-131 uptake at the left lower lung field (Fig. 3). The patient was then subjected to a high-dose radioiodine ablation $(150 \mathrm{mCi})$ on 10.5 .16 with posttherapy WBS which showed tracer accumulation in the

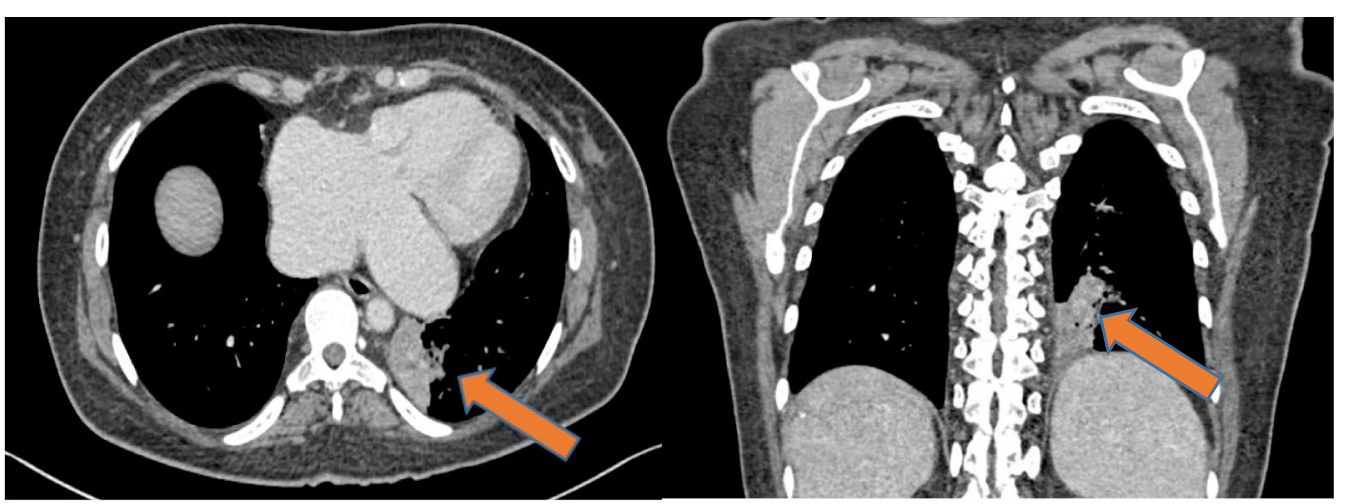

a) axial view

b) coronal view

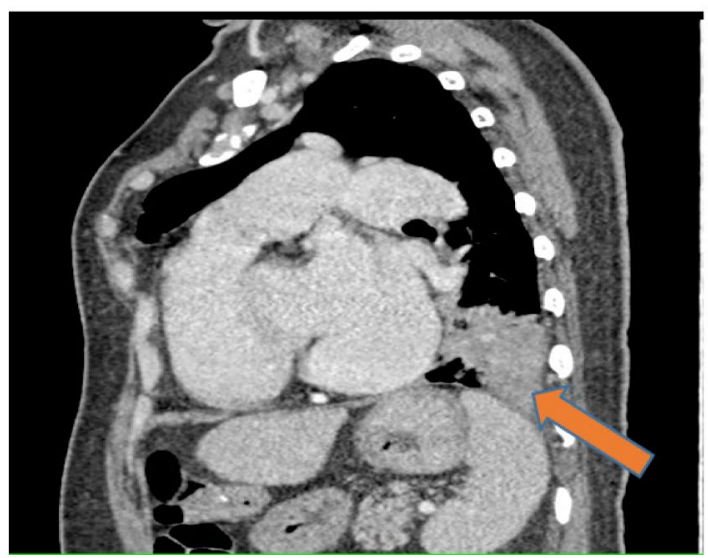

c) sagittal view

Fig. 1 CT thorax dated 2.12.14, a axial, b coronal and c sagittal views showing ill-defined heterogeneously enhancing mass at posterobasal segment of left lower lobe 


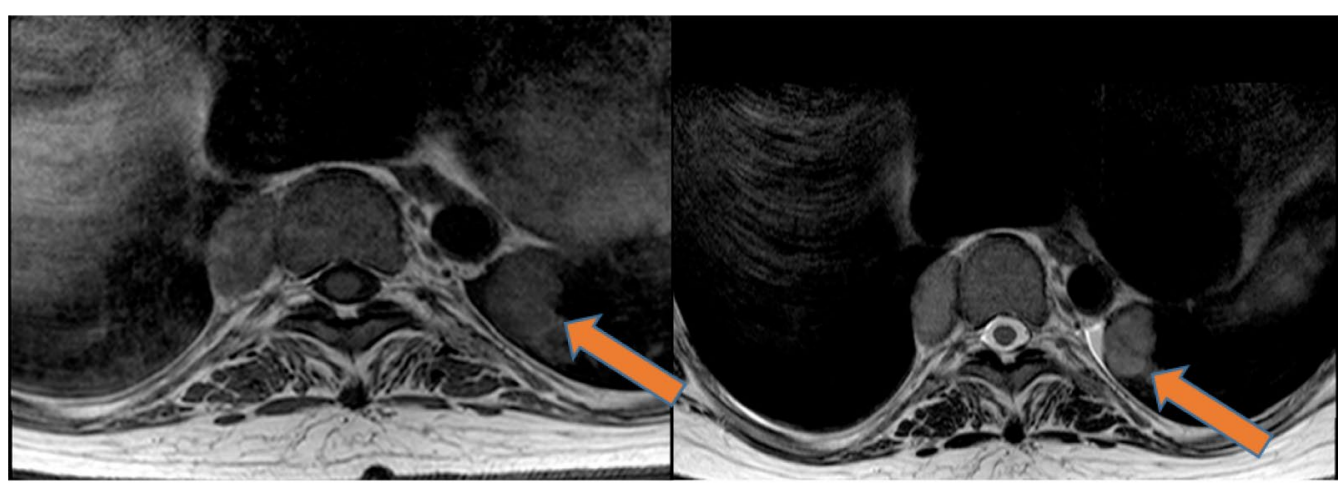

a) T1 weighted sequence

b) T2 weighted sequence

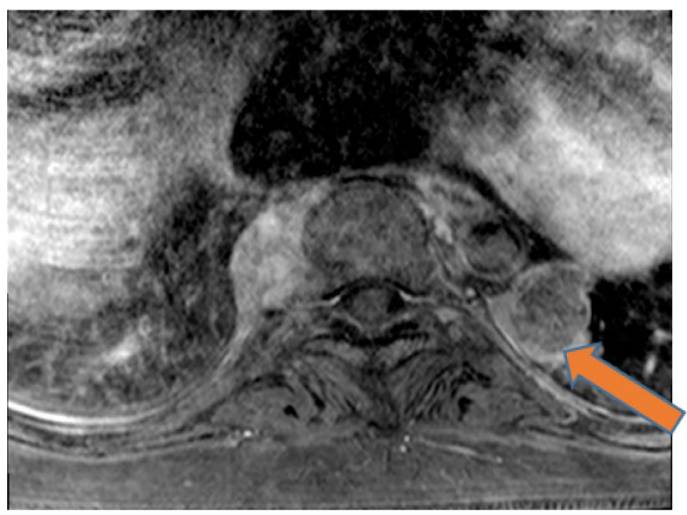

c) T1 weighted sequence with gadolinium

Fig. 2 Magnetic resonance imaging of patient (9.4.15); a T1 weighted, b T2 weighted and c T1 weighted with gadolinium showing lobulated lung lesion (arrow) at posterobasal segment of left lower lobe which is isointense to all muscle in all sequences and heterogeneously enhances post contrast. There is also a benign right paravertebral mass seen in the MRI study

thyroid bed and left lower lung field, similar to the initial diagnostic WBS. Another radioiodine ablation of $150 \mathrm{mCi}$ (total cumulative dose of $300 \mathrm{mCi}$ ) was given after a period of 6 months. Post-therapy WBS portrays the resolution of the previously seen uptake in the thyroid bed with persistent I-131 avidity at the left lower lung field (Fig. 4).

Her sequential stimulated and unstimulated serum $\mathrm{Tg}$ levels from August 2015 till January 2017 were undetectable (negative anti- $\operatorname{Tg} \mathrm{Ab}$ ) with only one recorded reading of $5.9 \mu \mathrm{g} / \mathrm{mL}$ (1.11.15) prior to her I-131 diagnostic WBS, which was attributed to the presence of remnant thyroid tissue (Table 1).

Written consent was obtained from the patient to report the case.

This case illustrates persistent I-131 uptake in the left lower lung field. However, the serum $\mathrm{Tg}$ was incessantly undetectable $(<0.04 \mu \mathrm{g} / \mathrm{ml})$ with negative anti-TgAb. Serial CT scans and MRI findings showed stable lung lesion in the posterior segment of left lower lobe that signifies pulmonary sequestration. Based on the clinical assessment, imaging findings and blood investigations, patient was no longer subjected to I-131 therapy and was presently on TSH suppression with Levothyroxine. After careful consideration of the risks and benefits, and lack of specialty for lung biopsy in our centre, patient opted against biopsy and surgical removal of the pulmonary lesion.

\section{Discussion}

Conscientious assessment of abnormal scans coupled with clinical assessment and biochemical correlation is essential to avert needless surgical intervention or ensuing ablation with high-dose radioiodine. False-positive I-131 uptake in WBS comprising the chest region has been reported in the literature. The aetiology encompasses acute respiratory infection, pulmonary tuberculosis, pulmonary aspergilloma, rheumatoid lung disease and bronchiectasis [1].

Pulmonary sequestration is an uncommon congenital anomaly of the lower respiratory tract which comprises a mass of lung tissue that is non-functioning [3]. The 


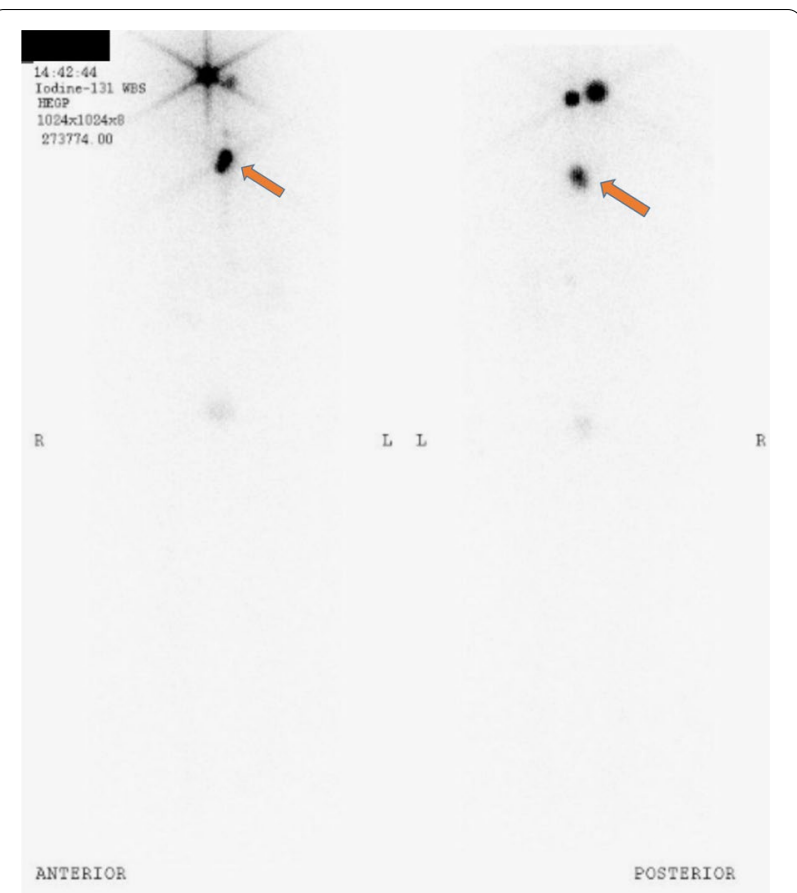

Fig. 3 -131 diagnostic whole-body scan (DxWBS) on 1.11.15 showed tracer uptake in the thyroid bed and left lower lung field (arrow)

incidence of pulmonary sequestration is 1 in 10,000 to 35,000 live births and represents 1 to $6 \%$ of the congenital abnormalities involving the lower airway $[2,3]$. In general, pulmonary sequestration can be classified into two forms. Intrapulmonary sequestration which is enclosed inside the visceral pleura of the adjoining lung accounts for $75 \%$ of the cases while extrapulmonary sequestration, which is situated externally with its own visceral pleura contributes the remaining 25\% [2]. Interestingly, almost two-thirds of intrapulmonary sequestration are found in the posterobasal segment of the left lower lobe [3] as demonstrated in this case.

The pathophysiology of pulmonary sequestration is not completely understood. The universally approved understanding is that it is derived during development from an accessory lung bud inferior to the normal lung buds [2, 3]. At the time of embryogenesis, the accessory lung bud acquires a separate vascular supply, independent from the normal developing tracheobronchial tree [3]. The airways of the lesion often reveal mucus accumulation, regions of inflammation, microcystic changes and distortion of lung parenchyma, while pathological examination demonstrates enlarged and thickened airspaces in the abnormal parenchyma [3].

The majority of patients are asymptomatic, and the disease is often diagnosed during routine medical examination or in recurrent bacterial lung infection affecting the lower lobe [5]. Symptoms are often non-specific consisting of chest pain, breathlessness, wheezing, fever, cough and recurrent infections [5], with infection being noted as the most common complication [3]. Other complications include creation of left to right shunt and haemorrhage, which can be fatal [3].

Imaging of pulmonary sequestration includes (i) arteriogram, which is vital in documenting systemic blood supply, (ii) chest X-ray, which usually shows homogenous dense mass in the thoracic cavity, (iii) ultrasound, which shows echogenic mass that is well defined or irregular, (iv) computed tomography (with accuracy of diagnosis of approximately $90 \%$ ), which shows solid homogenous or heterogenous mass and reveals anatomic position with vascular anatomy, and (v) magnetic resonance imaging, which demonstrates lung mass, systemic blood supply along with its venous drainage [2,5]. Management of lung sequestration in the asymptomatic patient is subject to controversy. Most authors advocate surgical resection of the lesions owing to the propensity for recurrent infection and possibility of haemorrhage [5].

Up to the present, only two cases of pulmonary sequestration causing false-positive findings in I-131 wholebody scan have been reported. The first case was reported by Jimenez-Bonilla et al. [6] in 2013 which showed I-131 uptake in a lesion at the right posterior costodiaphragmatic recess followed by Spinapolice et al. [7] in the ensuing year which reported a case of I-131 uptake of a nodular lesion in the upper mediastinum in between the aortic arc and pulmonary artery. The mechanism of I-131 uptake in pulmonary sequestration is not known. Examination of the surgical specimen from the case report by Spinapolice et al. [7] demonstrates a huge haemorrhagic infiltrate which might be responsible for I-131 fixation in the pulmonary sequestration.

In cases of discordant findings of low or undetectable serum $\mathrm{Tg}$ (with negative anti-TgAb) but positive I-131 WBS, single-photon emission computed tomography/ computed tomography (SPECT/CT) is strongly advocated whenever available. Study by Zilioli et al. [8] has shown that SPECT/CT has an additional value over planar whole-body scan in augmenting accuracy of diagnosis and lowering pitfalls and alters patient management by providing anatomical correlation and localization of radioiodine uptake.

\section{Conclusions}

Meticulous assessment of patient with suspected disease recurrence or metastasis in differentiated thyroid carcinoma is essential to avoid unnecessary I-131 ablation or surgical intervention. In this case, the positive I-131 imaging is associated with undetectable serum Tg 


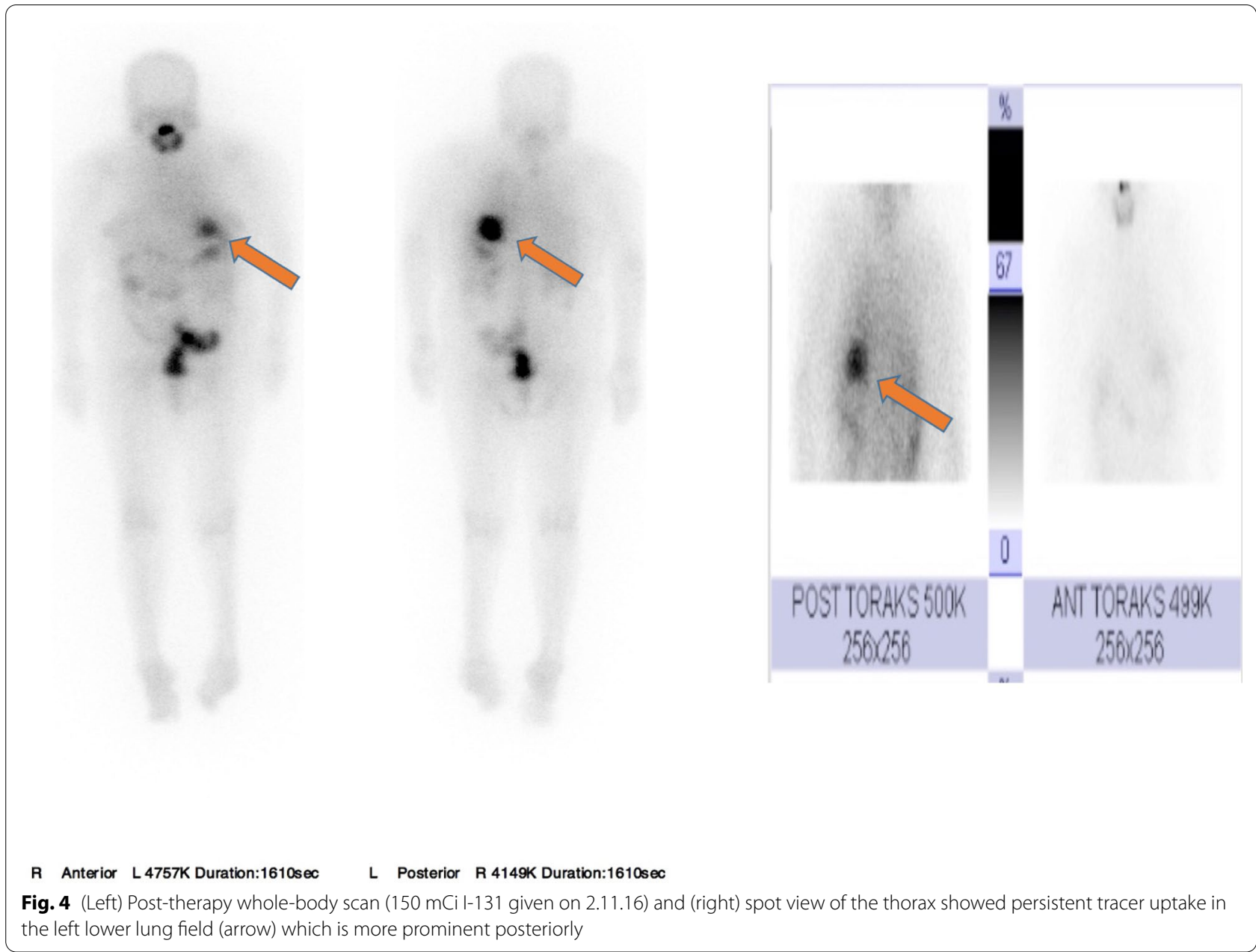

Table 1 Patient's stimulated thyroglobulin and anti-thyroglobulin antibody levels

\begin{tabular}{|c|c|c|c|c|c|c|c|c|c|}
\hline Date: & April 2011 & October 2011 & April 2012 & Feb 2013 & 1.11 .15 & 8.5 .16 & 16.8.16 & 31.10 .16 & 9.1 .17 \\
\hline TSH (mU/L) & 3.2 & 1.8 & 1.2 & 1.1 & 81.9 & $>100.0$ & 4.89 & $>100.0$ & 1.49 \\
\hline $\begin{array}{l}\text { Thyroglobulin (ug/ } \\
\text { mL) }\end{array}$ & 0.9 & $<0.04$ & $<0.04$ & 0.09 & 5.9 & $<0.04$ & $<0.04$ & $<0.04$ & $<0.04$ \\
\hline $\begin{array}{l}\text { Anti thyroglobulin } \\
\text { antibody (kIU/L) } \\
(<155)\end{array}$ & 14.9 & $<10.0$ & 11.3 & 10.9 & 13.8 & 26.4 & 12.9 & $<10.0$ & $<10.0$ \\
\hline Procedure & Post-operation & & & & Diagnostic WBS & Radioiodine & & & \\
\hline Ablation & & & Radioiodine ablation & & & & & & \\
\hline
\end{tabular}

(with negative anti-TgAb). Albeit uncommon, pulmonary sequestration should be taken into consideration as one of the differentials in false-positive findings of I-131 WBS.

\section{Abbreviations}

I-131: lodine-131; WBS: Whole-body scan; DTC: Differentiated thyroid carcinoma; Tg: Thyroglobulin; TSH: Thyroid-stimulating hormone; PS: Pulmonary sequestration.

\section{Acknowledgements}

We thank the Management of the Hospital Universiti Sains Malaysia (USM), Kubang Kerian Kelantan, Malaysia, for providing facilities for the study. Many thanks to co-authors for the contribution making this manuscript successfully produced. 
Authors' contributions

All the authors contribute in this case report. All authors read and approved the final manuscript.

\section{Funding}

Not applicable.

\section{Availability of data and material}

Not applicable.

\section{Declarations}

Ethic approval and consent to participate

Not applicable.

\section{Consent for publication}

Consent obtained.

\section{Competing interest}

The authors declare no competing interests.

\section{Author details}

${ }^{1}$ Department of Nuclear Medicine, Hospital Kuala Lumpur, 50300 Kampung Baru, Kuala Lumpur, Malaysia. ${ }^{2}$ Department of Nuclear Medicine, Radiotherapy and Oncology, School of Medical Sciences, Universiti Sains Malaysia, 16150 Kubang Kerian, Kelantan, Malaysia. ${ }^{3}$ Department of Radiology, School of Medical Sciences, Universiti Sains Malaysia, 16150 Kubang Kerian, Kelantan, Malaysia. ${ }^{4}$ Department of Nuclear Medicine, Radiotherapy and Oncology, Hospital Universiti Sains Malaysia, 16150 Kota Bharu, Kelantan, Malaysia. ${ }^{5}$ Department of Surgery, Hospital Raja Perempuan Zainab (II), 16150 Kota Bharu, Kelantan, Malaysia.

Received: 3 August 2021 Accepted: 10 February 2022

Published online: 18 February 2022

\section{References}

1. Oh J-R, Ahn B-C (2012) False-positive uptake on radioiodine whole-body scintigraphy: physiologic and pathologic variants unrelated to thyroid cancer. Am J Nucl Med Mol Imaging 2(3):362-385

2. Walker CM, Wu CC, Gilman MD, Godwin JD, Shepard JAO, Abbott GF (2014) The imaging spectrum of bronchopulmonary sequestration. Curr Probl Diagn Radiol 43(3):100-114. https://doi.org/10.1067/j.cpradiol.2014. 01.005

3. Oermann CM (2019) Bronchopulmonary sequestration, UpToDate. Available from https://www.uptodate.com/contents/bronchopulmonarysequestration. Accessed 15 July 2021.

4. Cooper DS, Doherty GM, Haugen BR, Kloos RT, Lee SL, Mandel SJ et al (2009) Revised American Thyroid Association management guidelines for patients with thyroid nodules and differentiated thyroid cancer. Thyroid 19(11):1167-1214. https://doi.org/10.1089/thy.2009.0110

5. Qian X, Sun Y, Liu D, Wu X, Wang Z, Tang Y (2015) Pulmonary sequestration: a case report and literature review. Int J Clin Exp Med 8(11):21822-21825

6. Jiménez-Bonilla JF, Bravo Z, Quirce R, Martínez-Rodríguez I, Banzo I, Gómez J et al (2013) An exceptional false-positive finding in the postsurgical I 131 whole body scan of a differentiated thyroid carcinoma caused by an extralobar pulmonary sequestration. J Clin Endocrinol Metab 98(5):1797-1798. https://doi.org/10.1210/jc.2013-1057

7. Spinapolice EG, Chytiris S, Fuccio C, Leporati P, Volpato G, Villani L et al (2014) Pulmonary sequestration: a (131)I whole body scintigraphy falsepositive result. Ann Nucl Med 28(7):683-687. https://doi.org/10.1007/ s12149-014-0838-5

8. Zilioli V, Peli A, Panarotto MB et al (2017) Differentiated thyroid carcinoma: incremental diagnostic value of ${ }^{131}$ I SPECT/CT over planar whole body scan after radioiodine therapy. Endocrine 56(3):551-559. https://doi.org/ 10.1007/s12020-016-1086-3

\section{Publisher's Note}

Springer Nature remains neutral with regard to jurisdictional claims in published maps and institutional affiliations.

\section{Submit your manuscript to a SpringerOpen ${ }^{\circ}$ journal and benefit from:}

- Convenient online submission

- Rigorous peer review

- Open access: articles freely available online

- High visibility within the field

- Retaining the copyright to your article 